\title{
The Proteasomal Subunits: Potential Therapeutic Targets for Cancer
}

\author{
Abdulfattah Salah Fararjeh* \\ PhD Program for Cancer Molecular Biology and Drug Discovery, College of Medical Science and Technology, Taipei Medical \\ University and Academia Sinica, Taiwan
}

\section{OPINION}

Without doubt, we can understand that cancer such as breast cancer is considered as a heterogeneous disease. It consists of several molecular subtypes and each subtype has a unique signaling pathways with a distinct growth and proliferation stimulatory. Throughout the last few decades, the information about the cellular process and signaling pathways that involved in cancer become clearer. These cellular processes and signaling pathways have been reported to affect the survival, proliferation, migration, invasion and apoptosis of the cancer cells [1-4]. Indeed, these signaling pathways frequently have cross talk between each other either direct or indirect to maintain breast cancer cell signaling on which able to respond to the external stimulators. Most of the researcher nowadays focusing on targeting these signaling pathways which can disrupt the internal cross talk signals in the cancer cell. However, another recent field that the scientist working on is the proteasome. The ubiquitin proteasomal system (UPS) is an important regulator for cell growth and cellular apoptosis. In cancer, the function and activities of proteasome are altered. For example, in apoptosis, several signaling pathways are included from releasing cytochrome C, activation Apaf-I containing activation of caspases apoptosome complex [5], all of these process by mitochondria. UPS system has an important role in regulating caspase and some of the apoptosis marker like BCL-2. For that, targeting proteasome could exert anticancer effect by inducing apoptosis and may also exert antitumor effect in-vivo.

\section{UBIQUITIN PROTEASOME SYSTEM AS A NOVEL TARGET FOR CANCER}

UPS system in cancer cells has an important role in maintaining the cells growth and survival by regulating several pathways. Furthermore, proteasomal activity is increased in cancer cells.
For that, several proteasome inhibitors have been developed which differentially controls the cellular proteins that involved in carcinogenesis or tumorigenesis process. The most popular one is MG-132 which is belongs to peptide aldehydes inhibitors, it also inhibits cathepsins and calpains. Another more potent and more specific to proteasome which has been developed is PS-341, boric acid peptide in inhibitor [6,7]. PS-341 has been investigated in-vitro using more than 60 cell lines including, human prostate, colorectal and breast cancer cell lines, and the result was clearly reflecting the potential of PS-341 as anticancer agent; it exerts cytotoxicity, cell cycle arrest, increase p21 and p27 and inhibiting NF-kB activities [8-10]. Overall, the mechanism for proteasome inhibitors in targeting cancer cells are varied; it could accumulate of growth inhibitory proteins, p21 and p27, or accumulation of BCL-2 pro-apoptotic family, or by inducing caspases pathways. Altogether, targeting proteasome is very important and recently it has been considered as a novel therapeutic strategy for cancer treatment.

More recently, several proteasomal subunits have been identified as biomarkers and novel therapeutic targets in malignancies; such as PSMD2 in lung and breast cancer [11,12], PSMD3 in breast cancer [13] and PSMB1 which was highly upregulated gene in bladder cancer [14]. Furthermore, increase proteasomal activity in tumor tissues compared to adjacent normal tissues were observed [15].

Modulation one or more of the proteasomal subunits by pharmacological intervention could be therapeutically advantageous. Knockdown some of the proteasomal subunits using siRNA technology have revealed to induce apoptosis, block cell cycle, inhibit cell proliferation in addition to inhibit tumor growth in some of the in-vivo studies. Because of the important role of the proteasomes in normal cellular process, non-specifically targeting might be toxic for the cells. Moreover, growing the knowledge on
Quick Response Code:

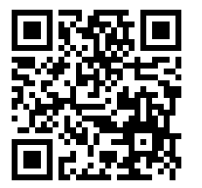

Address for correspondence: Abdulfattah Salah Fararjeh, PhD Program for Cancer Molecular Biology and Drug Discovery, College of Medical Science and Technology, Taipei Medical University and Academia Sinica, Taiwan

Received: August 01, 2019 Published: September 05, 2019

How to cite this article: Abdulfattah SF, The Proteasomal Subunits: Potential Therapeutic Targets for Cancer. 2019 - 1(2) OAJBS.ID.000104. DOI: 10.38125/OAJBS.000104 
the expression pattern and the role of the proteasome subunits in normal vs tumor cells could provide a rational foundation for the use of proteasome-targeting drugs.

\section{REFERENCES}

1. Hu C, Huang L, Gest C, Xi X, Janin A, et al. (2012) Opposite regulation by PI3K/Akt and MAPK/ERK pathways of tissue factor expression, cellassociated procoagulant activity and invasiveness in MDA-MB-231 cells. J Hematol Oncol 5: 16.

2. Citri A, Yarden Y (2006) EGF-ERBB signalling: towards the systems level. Nat Rev Mol Cell Biol 7(77): 505-516.

3. Xue G, Restuccia, Lan Q, Hynx D, Dirnhofer S, Hess D, et al. (2012) Akt/ PKB-mediated phosphorylation of Twist1 promotes tumor metastasis via mediating crosstalk between PI3K/Akt and TGF-beta signaling axes. Cancer Discov 2(3): 248-259.

4. Ascenzi P, Bocedi A, Marino M (2006) Structure function relationship of estrogen receptor alpha and beta: impact on human health. Mol Aspects Med 27(4): 299-402.

5. Bratton SB, MacFarlane M, Cain K, Cohen GM (2000) Protein complexes activate distinct caspase cascades in death receptor and stress-induced apoptosis. Exp Cell Res 256(1): 27-33.

6. Teicher BA, Anderson KC (2015) CCR $20^{\text {th }}$ anniversary commentary: In the beginning, there was PS-341. Clin Cancer Res 21(5): 939-941.

7. Lee KH, Jang AH, Yoo CG (2015) 17-Allylamino-17-Demethoxygeldanamycin and the Enhancement of PS-341-Induced Lung Cancer Cell Death by Blocking the NF-kappaB and PI3K/Akt Pathways. Am J Respir Cell Mol Biol 53(3): 412-421.
8. Hideshima T, Richardson P, Chauhan D, Palombella VJ, Elliott PJ, et al. (2001) The proteasome inhibitor PS-341 inhibits growth, induces apoptosis, and overcomes drug resistance in human multiple myeloma cells. Cancer Res 61(7): 3071-3076.

9. Cusack JC, Liu R, Houston M, Abendroth K, Elliott PJ et al. (2001) Enhanced chemosensitivity to CPT-11 with proteasome inhibitor PS341: implications for systemic nuclear factor-kappaB inhibition. Cancer Res 61(9): 3535-3540.

10. Zhang Y, Cheng J, Zhang J, Wu X, Chen F (2016) Proteasome inhibitor PS-341 limits macrophage necroptosis by promoting cIAPs-mediated inhibition of RIP1 and RIP3 activation. Biochem Biophys Res Commun 477(74): 761-767.

11. Matsuyama Y, Suzuki M, Arima C, Huang QM, Tomida S, et al. (2011) Proteasomal non-catalytic subunit PSMD2 as a potential therapeutic target in association with various clinicopathologic features in lung adenocarcinomas. Mol Carcinog 50(4): 301-309.

12. Li Y, Huang J, Zeng B, Yang D, Sun J, et al. (2018) PSMD2 regulates breast cancer cell proliferation and cell cycle progression by modulating p21 and p27 proteasomal degradation. Cancer Lett 430: 109-122.

13. Fararjeh AS, Chen LC, Ho YS, Cheng TC, Liu YR, (2019) Proteasome $26 \mathrm{~S}$ Subunit, non-ATPase 3 (PSMD3) Regulates Breast Cancer by Stabilizing HER2 from Degradation. Cancers 11(4): 527.

14. Jia Z, Ai X, Sun F, Zang T, Guan Y et al. (2015) Identification of new hub genes associated with bladder carcinoma via bioinformatics analysis. Tumori 101(1): 117-122.

15. Chen L, Madura K (2005) Increased proteasome activity, ubiquitinconjugating enzymes, and eEF1A translation factor detected in breast cancer tissue. Cancer Res 65: 5599-5606. 